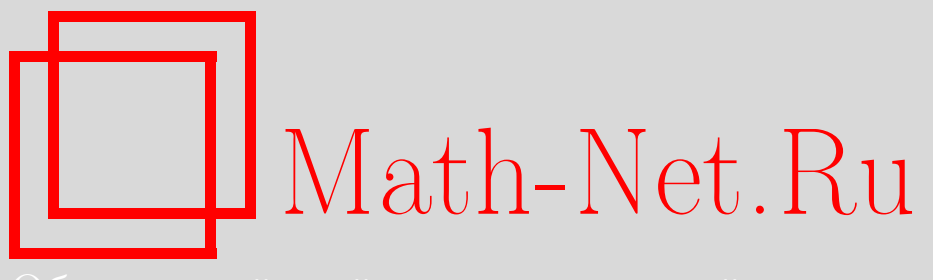

Общероссийский математический портал

Б. М. Гуревич, Динамические аспекты статистической физики в работах 
Р. Л. Добрушина, УМН, 1997, том 52, выпуск 2, 18-25

DOI: https://doi.org/10.4213/rm1584

Использование Общероссийского математического портала Math-Net.Ru подразумевает, что вы прочитали и согласны с пользовательским соглашением

http://www.mathnet.ru/rus/agreement

Параметры загрузки:

IP: 44.207 .124 .84

26 апреля 2023 г., 13:13:44 


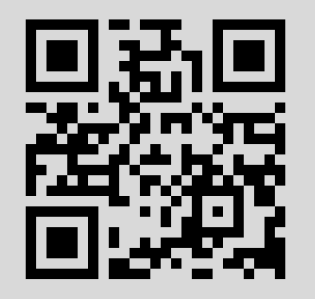




\section{ДИНАМИЧЕСКИЕ АСПЕКТЫ СТАТИСТИЧЕСКОЙ ФИЗИКИ \\ В РАБОТАХ Р. Л. ДОБРУШИНА}

\section{Б. М. ГУРЕВИч}

Статей, посвяшенных динамическим проблемам, у Р. Л. Добрушина не так уж много - около полутора десятков. Но он работал в этой области с большим увлечением, а полученные им результаты и, в еше большей степени, - его идеи и методы уже оказали и наверняка окажут в будущем существенное влияние на одно из важнейших направлений математической физики. 
Р. Л. Добрушин занимался задачами динамики с конца 70-х годов, вместе с несколькими своими учениками и сотрудниками. Естественно предположить, что его обращение к этой тематике было, по крайней мере отчасти, вызвано желанием применить к задачам неравновесной статистической физики "бесконечночастичньй" подход, основанньй на общем понятии гиббсовского случайного поля, которое было предложено им в конце 60-х годов и к тому времени уже успешно работало в равновесной теории. Последовательно переходя от одного круга задач к другому, логически связанному с предыдушим, он получил глубокие математические результаты, относяшиеся к трем центральньм вопросам неравновесной статистической физики: построению динамики, сходимости к равновесию и выводу гидродинамических уравнений.

Его первые работы, вьполненные совместно с Й. Фритцом, касались построения гамильтоновой динамики бесконечного числа взаимодействующих частиц. Речь идет, прежде всего, о теоремах сушествования и единственности решений бесконечной системы дифференциальных уравнений вида

$$
\dot{q}=v_{i}, \quad \dot{v}_{i}=-\sum_{j \neq i} \operatorname{grad}_{q_{i}} U\left(\left|q_{i}-q_{j}\right|\right), \quad i=1,2, \ldots,
$$

где $q_{i}, v_{i}-$ соответственно координата и скорость $i$-й частицы и $U$ - потенциал попарного взаимодействия.

Впервые такие системы рассматривали Т. Харрис [1] и О. Лэнфорд [2], которые, в частности, заметили, что при нетривиальном взаимодействии решение задачи Коши не обязано существовать, а тем более - быть единственным для всех начальных условий. Это объясняется возможностью "гравитационного коллапса", когда за конечное (и даже сколь угодно малое) время бесконечно много частиц попадает в ограниченную часть пространства. В [2] было описано некоторое множество $X$ “допустимых" начальных условий, обладающее тем свойством, что через каждую точку $x=\left\{\left(q_{i}, v_{i}\right), i=1,2, \ldots\right\} \in X$ проходит единственное решение $x(t)=\left\{\left(q_{i}(t), v_{i}(t)\right), i=1,2, \ldots, t \geqslant 0\right\}, x(0)=x$ и $x(t) \in X$ при всех $t \geqslant 0$. В результате оказьвается возможным определить на $X$ полугруппу преобразований $S^{t}: x(0) \mapsto x(t), t \geqslant 0$, которую часто называют динамической системой статистической механики или просто динамикой.

В [2] предполагалось, что потенциал $U$ - это гладкая на всей оси функция, а в [1] - что $U$ есть, формально говоря, $\delta$-функция. В обоих случаях сила, действуюшая на каждую из двух сближаюшихся частиц, остается ограниченной, что технически удобно, так как позволяет контролировать поведение траекторий в фазовом пространстве, но, с физической точки зрения, неестественно.

Описанный дефект сумел устранить Я.Г. Синай [3], [4], которьй построил динамику для некоторого класса потенциалов, гладких вне нуля и стремяшихся к бесконечности в нуле. Однако в его работах, как и в работах его последователей [5], [6], не давалось явного описания множества допустимых начальных условий, а лишш доказывалось, что на нем сосредоточено любое равновесное распределение $G_{U, \mu, v, \beta}$, отвечаюшее потенщиалу $U$. Такое распределение есть гиббсовская мера в смысле Добрушина-Лэнфорда-Рюэля с формальным гамильтонианом

$$
H_{U, \mu, v, \beta,}\left(q_{1}, v_{1}, q_{2}, v_{2}, \ldots\right)=\frac{1}{2} \beta \sum_{i}\left[\mu+\left(v_{i}-v\right)^{2}\right]+\beta \sum_{i<j} U\left(\left|q_{i}-q_{j}\right|\right),
$$


где $\mu, v, \beta$ - параметры, связанные с законами сохранения массы, импульса и энергии соответственно. Такая мера инвариантна относительно динамики $\left\{S^{t}\right\}$, и тем самым ее временна́я эволюция тривиальна. Что же касается временно́й эволюции неравновесных распределений, представляющей принципиальньй интерес, то говорить о ней в данной схеме затруднительно, так как отсутствие явного описания множества $X$ не позволяет судить о том, какие из этих распределений (кроме абсолютно непрерывных относительно $\left.G_{U, \mu, v, \beta}\right)$ сосредоточены на $X$.

Р. Л. Добрушину и Й. Фритцу [7], [8] удалось преодолеть эту трудность в размерностях 1 и 2. С помошью тонких рассуждений, основанных на законе сохранения энергии, они дали явное и достаточно простое описание множества, на котором определена динамика, и показали, что на нем сосредоточены гиббсовские распределения, отвечающие не только потенциалу $U$, но и любому потенциалу $\widetilde{U}$ конечного радиуса, удовлетворяющему при некоторых $a, b=$ const неравенству $a+b \widetilde{U} \geqslant U$. Тем самым, появилась возможность определить временну́ю эволюцию неравновесных гиббсовских мер, отвечаюших широкому классу физически естественных взаимодействий. Имея в виду это обстоятельство, иногда говорят, что в [7], [8] построена неравновесная динамика.

Следуюшая проблема, привлекавшая внимание Р. Л. Добрушина, связана с обоснованием постулата Больцмана-Гиббса (в классической статистической физике), согласно которому система большого числа частиц, находящаяся в состоянии равновесия, должна описьваться равновесным распределением Гиббса. Существует подход к этой проблеме, основанньй на вариационном принципе Гиббса и не выходящий за рамки равновесной теории. Но естественно стремиться к другому, гораздо более удовлетворительному обоснованию, использующему тот факт (если бы его удалось доказать), что любая вероятностная мера общего вида, заданная на фазовом пространстве системы частиц, под действием динамики сходится в том или ином смысле к одному из равновесных распределений. В отличие от вариационного принципа, такой результат не может быть получен для системы с фиксированным числом частищ, и бесконечночастичньй подход Добрушина представляется здесь совершенно естественным.

Однако задача чрезвычайно трудна и на современном уровне знаний о динамике вряд ли может быть решена для сколько-нибудь обших взаимодействий. Поэтому Р.Л. Добрушин предложил рассмотреть вырожденные модели, описываюшие, например, идеальньй (свободньй) газ или систему твердых стержней, свободно движущихся вдоль оси и обменивающихся скоростями при столкновении. Хотя взаимодействие в этих системах в максимально возможной степени вырождено, даже в случае первой из них нельзя утверждать, что оно полностью отсутствует: суррогатом взаимодействия служит условие неразличимости частиц, без которого система распалась бы в прямое произведение бесконечного числа систем с одной степенью свободы.

Чтобы описать результаты, полученные Р.Л. Добрушиньм и его соавторами К. Болдригини и Ю.М. Суховьм [9]-[11], напомним, что равновесное распределение, отвечающее нетривиальному потеншиалу взаимодействия $U$, обьчно зависит еще от одного векторного и двух скалярных параметров (см. приведенную вьше формулу для гамильтониана), связанных с асимптотически-аддитивными первыми интегралами конечных систем с потенциалом взаимодействия $U$ : числом частиц, полной энергией и полным импульсом (см. по этому поводу [12]). У вырожденных же систем семейство аддитивных интегралов гораздо богаче: например, в случае свободного газа 
в пространстве $\mathbb{R}^{d}$ для любого $d_{1}$ и любой функции $f: \mathbb{R}^{d} \rightarrow \mathbb{R}^{d_{1}}$ сумма $\sum_{i=1}^{n} f\left(v_{i}\right)$ служит первьм интегралом системы с $n$ степенями свободы и, следовательно, порождает аддитивный интеграл. Полная энергия и полный импульс соответствуют здесь частным случаям, когда $d_{1}=1, f(v)=v^{2} / 2$ (при $d>1$ имеется в виду скалярный квадрат) и $d_{1}=d, f(v)=v$. Поэтому равновесное распределение $G_{\mu, Q}$ зависит от скалярного параметра $\mu$ (плотности частиц в координатном пространстве) и функционального параметра $Q$, задающего распределение в пространстве скоростей (при фиксированных координатах скорости всех частиц независимы).

Если $\mathrm{P}$ - вероятностная мера, заданная на фазовом пространстве бесконечной системы (которое можно отождествить с множеством $X$, где определена динамика), то ее временна́я эволюция описывается семейством вероятностных мер $\mathrm{P}_{t}, t \geqslant 0$, где $\mathrm{P}_{t}(A)=\mathrm{P}\left(S_{t}^{-1} A\right)$ для любого измеримого множества $A \subset X$. В работах [9]-[11] были найдены достаточные условия слабой сходимости $\mathrm{P}_{t} \rightarrow G_{\mu, Q}$ при $t \rightarrow \infty$. О тех из них, которые носят технический характер, мы говорить не будем, но два условия являются принципиальными. Первое формулируется в терминах одночастичной корреляционной (моментной) меры $K_{\mathrm{P}}^{(1)}$, отвечающей вероятностной мере Р. По определению $K_{\mathrm{P}}^{(1)}$ - это мера на фазовом пространстве $X^{(1)}$ одночастичной системы, которая задается равенством $K_{\mathrm{P}}^{(1)}(B)=\mathrm{E}_{\mathrm{P}} N^{(1)}(B)$, где $N^{(1)}(B)$ - число точек из бесконечной случайной конфигурации, принадлежащих измеримому множеству $B \subset X^{(1)}$ (ясно, что $N^{(1)}(\cdot)$ - случайная мера со значениями в $\left.\mathbb{Z}^{+}\right)$, и $\mathrm{E}_{\mathrm{P}}$ - математическое ожидание, т.е. интеграл по $\mathrm{P}$ (подобным же образом определяется $n$-частичная корреляционная мера при любом $n>2$ ). В частном случае, когда мера Р трансляционно-инвариантна, требуется, чтобы ее коррелящионная мера $K_{\mathrm{P}}^{(1)}$ совпадала с коррелящионной мерой равновесного распределения $G_{\mu, Q}$. Это условие является не только достаточным, но и необходимым, оно выделяет то из равновесных распределений, к которому сходится $\mathrm{P}_{t}$, если сходимость к одному из них имеет место. В общем случае достаточно, чтобы мера $K_{\mathrm{P}, t}^{(1)}$, получающаяся из $K_{\mathrm{P}}^{(1)}$ под действием свободной динамики в $X^{(1)}$ (т.е. $K_{\mathrm{P}, t}^{(1)}(B)=K_{\mathrm{P}}^{(1)}\left(T_{t}^{-1} B\right)$, где $\left.T_{t}(q, v)=(q-t v, v)\right)$, в естественном смысле сходилась при $t \rightarrow \infty$ к корреляционной мере равновесного распределения $G_{\mu, Q}$.

Второе из упомянутых важных условий состоит в том, что события, определяемые поведением бесконечной случайной конфигурации в двух областях координатного пространства, расположенных на расстоянии $r$ друг от друга, при $r \rightarrow \infty$ становятся асимптотически независимыми. Это аналог известного в теории стационарных случайных процессов условия сильного перемешивания Розенблатта, и авторы выражают сомнение в том, что без какого-либо условия такого типа можно обойтись (эта точка зрения до сих пор не опровергнута).

Стоит отметить, что в [9]-[11] мера Р вовсе не предполагалась гиббсовской и авторы использовали гиббсовские меры с достаточно регулярными потенциалами лишш как примеры, для которых выполняются наложенные ими условия.

Несмотря на то, что за время, прошедшее после опубликования упомянутых работ, каких-либо существенных сдвигов в данной области не произошло, они не потеряли своего значения: в этих работах были введены важные понятия, связанные с проблемой сходимости к равновесию, и впервые было показано, что сушествуют системы, для которых эта проблема может быть решена на математическом уровне строгости.

Однако изучение сходимости к равновесию было для Р. Л. Добрушина хоть и не- 
обходимьм, но все же промежуточным этапом на пути к более важной цели - выводу гидродинамических уравнений из динамики частиц. К этой проблематике он подошел в самом начале 80-х годов и не расставался с ней более десяти лет, несмотря на интенсивные занятия другими задачами. Точка зрения, что такой вывод возможен, высказывалась в физической литературе уже давно (достаточно вспомнить работы Н.Н. Боголюбова и его школы [13], [14]), но математическую формулировку, по-видимому, впервые предложил в середине 50-х годов Ч. Морри [15], [16]. Р. Л. Добрушин и его соавторы в этой связи часто ссылались на Морри, однако последний, в отличие от них, рассматривал конечные системы с растущим числом степеней свободы, и приписываемый ему подход было бы более справедливо считать интерпретацией.

Так или иначе, это подход состоит в следующем. Предполагается, что начальное распределение вероятностей $\mathrm{P}$ на фазовом пространстве $X$ бесконечной системы локально однородно по пространству в том смысле, что оно почти переходит в себя при пространственных сдвигах на расстояния, много меншшие некоторого $\varepsilon^{-1}$, где $\varepsilon$ - мальй параметр. Как правило, достаточно требовать лишь сушествования и локальной однородности одночастичной корреляционной функции $k_{\mathrm{P}}^{(1)}(q, v)$, которая определяется как плотность одночастичной корреляционной меры $K_{\mathrm{P}}^{(1)}$ (см. выше) относительно меры Лебега (и, быть может, двухчастичной корреляционной функции $k_{\mathrm{P}}^{(2)}(q, v)$, которая определяется аналогичным образом по $\left.K_{\mathrm{P}}^{(2)}\right)$. Это приводит к рассмотрению семейства начальных распределений $\left\{\mathrm{P}^{\varepsilon}, \varepsilon>0\right\}$, сосредоточенных на $X$, а локальная однородность начального распределения выражается в виде равенства $\lim _{\varepsilon \rightarrow 0} k_{\mathrm{P} \varepsilon}^{(1)}(q / \varepsilon, v)=f_{0}(q, v)$, где $f_{0}$ - гладкая функция.

Требуется установить, что локальная однородность не исчезает с течением времени, т.е. что при любом $t>0$ существует предел $f(q, v, t)=\lim _{\varepsilon \rightarrow 0} k_{\mathrm{P}_{t / \varepsilon}^{\varepsilon}}^{(1)}(q / \varepsilon, v)$, гладко зависяший от $q, t$ и такой, что $f(q, v, 0)=f_{0}(q, v)$. Функция $f$, описывающая состояние непрерывной среды, должна удовлетворять некоторому уравнению, имеюшему смысл гидродинамического уравнения Эйлера, но его конкретньй вид может зависеть от рассматриваемой модели. Вот как, например, оно вьглядит для системы твердых стержней длины $l$ (это известное в физике уравнение Перкуса, впервые вьведенное в [20] на математическом уровне строгости):

$$
\begin{aligned}
& \frac{\partial}{\partial t} f(q, v, t)+v \frac{\partial}{\partial q} f(q, v, t) \\
& \quad+l \frac{\partial}{\partial q}\left\{f(q, v, t)\left[1-l \int_{-\infty}^{\infty} f(q, \xi, t) d \xi\right]^{-1} \int_{-\infty}^{\infty} l \eta(v-\eta) f(q, \eta, t) d \eta\right\}=0 .
\end{aligned}
$$

Вместо корреляционных мер можно рассматривать интегралы по этим мерам от числа частиц, скорости и энергии и получать, как это обьчно и делается в гидродинамике, уравнения, которым должны удовлетворять пределы (при $\varepsilon \rightarrow 0$ ) этих величин.

В описанной схеме явно присутствуют две временны́е шкалы - микроскопическая и макроскопическая, связанные коэффициентом пропорциональности $\varepsilon$ : если $t$ - макроскопическое (гидродинамическое) время, то $t / \varepsilon-$ микроскопическое (динамическое время). Точно так же связаны микроскопическая и макроскопическая пространственные шкалы (последнее обстоятельство соответствует представлению о том, что частица жидкости - это “физически” бесконечно малый объем, содержаший все еще очень 
большое число молекул). При этом потенциал взаимодействия и плотность частиц (в микрошкале) остаются постоянньми. Произведенньй в таких условиях переход к пределу при $\varepsilon \rightarrow 0$ называют гидродинамическим предельным переходом.

При выводе гидродинамических уравнений Морри предполагал наличие у динамики частиц ряда свойств, которые ему не удалось проверить. Р. Л. Добрушин считал это вполне закономерньм, поскольку согласно его точке зрения для строгого вьвода таких уравнений нужна гораздо более глубокая, чем имеющаяся сегодня, информация о динамике. В частности, необходимо уметь доказьвать сходимость к равновесию для достаточно богатого семейства начальных распределений. Последнее уже было сделано для нескольких вырожденных моделей в предыдуших работах Р. Л. Добрушина. Для этих моделей (к упомянутьм вьше необходимо присоединить систему гармонических осцилляторов на одномерной решетке и модели стохастической динамики) ему (в соавторстве с рядом коллег, см. [17]-[24]) удалось реализовать свою программу, причем не только в отношении уравнения Эйлера, описьваюшего среду с нулевой вязкостью, но и уравнений более высокого порядка, в том числе аналогов уравнения Навье-Стокса, которое он рассматривал как поправку первого порядка по $\varepsilon$ к приближенному описанию динамики, даваемому уравнением Эйлера. При этом он неизменно подчеркивал (и это было отражено даже в заголовках статей), что речь идет лишш о карикатуре на гидродинамику, которая, однако, как всякая карикатура, должна отражать какие-то характерные черты оригинала.

Описание деятельности Р. Л. Добрушина в области неравновесной статистической физики было бы неполным без упоминания его работы [25], посвященной кинетическому уравнению Власова, в которой он, сушественно обобшив постановку задачи, доказал, в частности, теорему единственности для решений этого уравнения.

В заключение несколько слов, характеризуюших Р. Л. Добрушина уже не только как математика, и несколько отрывочных воспоминаний о нем.

Впервые я увидел Р. Л. Добрушина в конце 50-х годов, когда он, будучи сотрудником кафедры теории вероятностей МГУ, читал нам, студентам 3-го курса, обязательные лекции по этому предмету. Но еше за год с лишним до этого в коридорах можно было часто услышать это имя, ставшее почти легендарным из-за необычно смелого выступления Р. Л. на факультетском “активе”. Его лекции отличались исключительной ясностью, чувствовалось, что он заранее продумьвал не только содержание, но и форму изложения. При этом ему удавалось наряду с вешами абсолютно необходимыми рассказывать и кое-что новое. Так, он познакомил нас с основными понятиями теории информации, которой в то время сам начинал заниматься.

Однако, будучи выдаюшимся ученым, Р. Л. Добрушин интересовался не только наукой. Вспоминается небольшой эпизод, относящийся к 60-м годам, когда в Фергане проходила конференщия по предельным теоремам теории вероятностей. За день до ее начала, уже ближе к вечеру, группа участников после многочасового автобусного переезда из Ташкента достигла наконец общежития местного пединститута. Многие уже собрались отдохнуть, когда в коридоре раздался бодрьй голос Р. Л.: “Давайте возьмем такси и поедем смотреть пустьню!" Сочетание такси и пустыни производило сильное впечатление, но по сушеству он был прав: в остававшееся до темноты время никакой другой транспорт не годился, а ему не терпелось увидеть новые места, тем более, что предстояла интенсивная работа, и лучшего момента могло не представиться. Этот живой и не убывавший с годами интерес ко всему для него новому Р. Л. 
сохранил до конца жизни. В последнее время, получив возможность свободно ездить по миру, он побывал во многих странах; за несколько месяцев до смерти, уже будучи тяжело больньм и зная об этом, совершил научную поездку в Сингапур, о которой потом с увлечением рассказывал.

В течение многих лет Р. Л. Добрушин был одним из руководителей семинара по математическим вопросам статистической физики, работавшего на мехмате МГУ. В свободной атмосфере, обычно царившей на заседаниях, после докладов, а часто и по ходу их возникали дискуссии, касавшиеся возможных интерпретаций сказанного или правдоподобности тех или иных гипотез. Конечно, это сильно замедляло работу, но приносило подчас даже больше пользы, чем сами доклады. Р. Л. Добрушин был едва ли не самьм активным участником таких дискуссий, неизменно демонстрируя математическую интуицию и проницательность. Впрочем, иногда он оказьвался в чем-то неправ, и тогда, произнеся свою обычную для таких случаев фразу "Глупость сказал", он садился на место. В нем не было ни капли заносчивости или снобизма, и он всегда был готов признать свою ошибку, если убеждался, что совершил ее. Но так же откровенно он высказьвал свои твердо устоявшиеся взгляды по научньм и иным вопросам, не очень заботясь о том, чтобы это устраивала каждого. Из всех, кого я знал, к нему, быть может, в наибольшей мере подходит характеристика "естественный человек" настолько естественным и непосредственным казалось его восприятие окружающего. Однако это вовсе не мешало ему в специфической сфере деятельности - организационных и административных делах - проявлять и гибкость, и дипломатичность, если это требовалось для достижения необходимого результата. И эти качества, дополнявшие его большой научньй потенциал, благотворно сказывались на работе возглавлявшейся им лаборатории в Институте проблем передачи информации, которая теперь по праву носит его имя.

\section{СПИСОК ЛИТЕРАТУРЫ}

[1] Harris T. E. Diffusion with "collisions" between particles // J. Appl. Probab. 1965. V. 2. № 2. P. 323-338.

[2] Lanford O.E. The classical mechanics of one-dimensional systems of infinitely many particles. 1. An existence theorem // Comm. Math. Phys. 1968. V. 9. № 3. P. 176-191.

[3] Синай Я.Г. Построение динамики в одномерных системах статистической механики // ТМФ. 1972. Т. 11. № 2. С. 248-258.

[4] Синай Я. Г. Построение кластерной динамики для динамических систем статистической механики // Вестн. МГУ. Сер. матем., мех. 1974. Т. 29. № 3. С. 152-158.

[5] Земляков А.Н. Построение динамики в одномерных системах статистической физики в случае нефинитных потенциалов // УМН. 1973. Т. 28. №1. С. 239-240.

[6] Presutti E., Pulvirenti M., Tirozzi B. Time evolution of infinite classical systems with singular, long range, two body interactions // Comm. Math. Phys. 1976. V. 47. № 1. Р. 81-95; Презутти Э., Пульвиренти М., Тироцци Б. Эволюция во времени бесконечных классических систем с сингулярным дальнодействующим парным потенциалом // Гиббсовские состояния в статистической физике. М.: Мир, 1978. С. 219-240.

[7] Dobrushin R. L., Fritz J. Non-equilibrium dynamics of one-dimensional infinite particle systems with a singular interaction // Comm. Math. Phys. 1977. V. 55. № 3. P. 275-292.

[8] Dobrushin R. L., Fritz J. Non-equilibrium dynamics of two-dimensional infinite particle systems with a singular interaction // Comm. Math. Phys. 1977. V. 57. № 1. P. 67-81.

[9] Dobrushin R. L., Sukhov Yu.M. On the problem of the mathematical foundation of the Gibbs postulate in classical statistical mechanics // Lecture Notes in Phys. 1978. V. 80. P. $325-340$. 
[10] Добрушин Р. Л., Сухов Ю. М. Временна́я асимптотика для некоторых вырожденных моделей эволюции систем с бесконечным числом частиц // Итоги науки и техники. Соврем. пробл. матем. Т. 14. М.: ВИНИТИ, 1979. С. 147-250.

[11] Boldrighini C., Dobrushin R. L., Sukhov Yu. M. The Asymptotics for Some Degenerate Models of the Evolution of Infinite Particle Systems // Preprint: Università degli Studi di Camerino, 1980.

[12] Гуревич Б. М. О гиббсовских случайных полях, инвариантных относительно бесконечночастичной гамильтоновой динамики // ТМФ. 1992. Т. 90. № 3. С. 424-459.

[13] Боголюбов Н.Н. Проблемы динамической теории в статистической физике. М.-Л.: ОГИЗ. Гостехиздат, 1946 (см. также Боголюбов Н. Н. Избранные труды, 2. Киев: Наукова думка. 1970. С. 99-196).

[14] Зубарев Д. Н. Неравновесная статистическая термодинамика. М.: Наука, 1973.

[15] Morrey Ch. B. On the derivation of the equations of hydrodynamics from statistical mechanics // Proc. Nat. Acad. Sci. USA. 1954. V. 40. № 5. P. 317-322.

[16] Morrey $\mathrm{Ch}$. B. On the derivation of the equations of hydrodynamics from statistical mechanics // Comm. Pure Appl. Math. 1955. V. 8. № 2. P. 279-326.

[17] Болдригини К., Добрушин Р. Л., Сухов Ю. М. Гидродинамика одномерных твердых стержней // УМН. 1980. Т. 35. № 4. С. 252-253.

[18] Dobrushin R.L., Siegmund-Schultze R. The hydrodynamic limit for systems of particles with independent evolution // Math. Nachr. 1982. V. 105. № 1. P. 199-224.

[19] Добрушин Р.Л. Гидродинамический предельный переход. Некоторые карикатуры // Взаимодействующие марковские процессы и их применение к математическому моделированию биологических систем. Пущино, 1982. С. 7-20.

[20] Boldrighini C., Dobrushin R.L., Sukhov Yu. M. One dimensional hard rod caricature of hydrodynamics // J. Statist. Phys. 1983. V. 31. № 3. P. 577-615.

[21] Dobrushin R.L., Pellegrinotti A., Sukhov Yu. M., Triolo L. One-dimensional harmonic lattice caricature of hydrodynamics // J. Statist. Phys. 1986. V. 43. № 3/4. P. 571-608.

[22] Dobrushin R.L., Pellegrinotti A., Sukhov Yu. M., Triolo L. One-dimensional harmonic lattice caricature of hydrodynamics: second approximation // J. Stat. Phys. 1988. V. 52. № 1/2. P. 423-439.

[23] Dobrushin R. L. Caricature of hydrodynamics // Proc. IX Intern. Congr. Math. Phys. Adam Higler. Bristol. 1989. P. 117-132.

[24] Dobrushin R. L., Sokolovskii S. Higher order hydrodynamic equations for a system of independent random walks // Random walks, Brownian motion and interacting particle systems. Boston: Birkhauser, 1990. P. 231-254.

[25] Добрушин Р. Л.Уравнение Власова // Функц. анализ. и его прилож. 1979. Т. 13. № 2. C. $48-58$. 